\title{
ESQUEMA FLUX-SPLINE APLICADO A PROBLEMAS DIFUSIVOS TRIDIMENSIONAIS EM REGIME PERMANENTE
}

\author{
Paulo César Oliveira ${ }^{1}$
}

\begin{abstract}
RESUMO
Este trabalho teve por finalidade apresentar um esquema de discretização mais eficiente que o tradicional esquema de diferenças centrais, denominado Flux-Spline, a fim de simular, numericamente, problemas tridimensionais governados por difusão. A eficiência do esquema proposto foi avaliada por meio da resolução de problemas-teste com solução analítica conhecida. Verificou-se que tal esquema possui características adequadas de precisão e, portanto, uma opção recomendável para a solução de problemas difusivos tridimensionais.
\end{abstract}

Palavras-chave: difusão, volumes finitos, transferência de calor, métodos numéricos

\section{FLUX-SPLINE SCHEME APPLIED TO STEADY STATE TRIDIMENSIONAL DIFFUSIVE PROBLEMS}

\begin{abstract}
This objective of this research was to present a discretization scheme more efficient than the traditional central difference scheme, called Flux-Spline, in order to simulate tridimensional problems governed by diffusion. The efficiency of the proposed scheme was evaluated by means of the resolution of two test problems with a known analytical solution. The results showed a discretization scheme with adequate characteristics of accuracy that is, therefore, a recommendable option for the solution of tridimensional diffusive problems.
\end{abstract}

Key words: diffusion, finite volumes, heat transfer, numerical methods

\section{INTRODUÇÃO}

Processos físicos governados por difusão pura aparecem em fenômenos de interesse prático, como escoamento em meios porosos, tais como solos agrícolas e materiais biológicos, no processamento de alimentos, na condução de calor em sólidos, em processos metalúrgicos contendo mudanças de fase, nos escoamentos potenciais ou completamente desenvolvidos em dutos, na difusão e transferência de massa.

Embora em alguns casos particulares se conheça uma solução analítica para as equações diferenciais parciais que governam tais fenômenos, a presença de geometrias não regulares e a ausência de linearidade devido à variação das propriedades do meio ou a existência de condições de contorno particulares, impossibilitam a solução desses problemas com os métodos analíticos atualmente conhecidos, tornando obrigatória a utilização de métodos numéricos.

Exemplos de aplicação do método de volumes finitos, usando-se o tradicional esquema de discretização em problemas bidimensionais de secagem, são encontrados no trabalho de França \& Fortes (1991) que trata da simulação bidimensional de secagem de milho em leito fixo, e em Oliveira et al. (1991) que

\footnotetext{
${ }^{1}$ Professor, Doutor, Departamento de Engenharia Rural, Centro Agropecuário, UFES, CP 16, CEP 29500 - 000, Alegre, ES, Fone: (027) 5521400 - Ramal: 233, Fax: (027) 552 2622, E-mail :caufes@ npd.ufes.br
} 
abordaram a secagem de milho acoplada à convecção natural em uma das faces do silo.

Com o objetivo de se reduzir o esforço computacional, pode ser empregado, nesses casos, um esquema de discretização denominado Flux-Spline que, como mostram os trabalhos de Varejão (1979), Nieckele (1985), Varejão et al. (1991a), Varejão et al. (1991b), e Oliveira (1997) tem desempenho superior aos esquemas tradicionais utilizados por Patankar (1980) para problemas difusivos e convectivos bidimensionais em regime permanente. Este trabalho pretende mostrar que tal superioridade se mantém para casos difusivos tridimensionais, podendo-se obter resultados adequados em termos de erro, com malhas relativamente grosseiras.

\section{MATERIAL E MÉTODOS}

\section{Desenvolvimento do Esquema Flux-Spline}

Nesta seção, o esquema será detalhado para o caso unidimensional em regime permanente, a partir do qual será gerada a formulação multidimensional. A discretização é baseada sobre uma formulação de volumes de controle.

\section{Difusão Unidimensional em Regime Permanente}

A equação de governo para problemas difusivos tridimensionais em regime permanente é:

$$
\frac{\partial J x}{\partial x}+\frac{\partial J y}{\partial y}+\frac{\partial J z}{\partial z}=S^{\phi}
$$

em que $J$ é o fluxo difusivo nas direções x, y e z e $S$ f o termo fonte.

Para um caso unidimensional:

$$
\frac{\partial J}{\partial x}=S^{\phi}
$$

O termo fonte $S^{f}$ é linearizado ao longo do domínio da variável dependente $f$, na forma $S^{f}=S_{c}+S_{p} . f\left(\right.$ Patankar, 1980) sendo $S_{c}$ a parte constante do termo fonte e $S_{p}$ função da variável dependente f. O fluxo difusivo $J$, na direção x, é dado por:

$$
J=-\mathrm{K} \cdot \frac{\partial \phi}{\partial x}
$$

em que $\mathrm{K}$ representa o coeficiente de difusão, podendo ser ou não função da variável dependente $f$.

Integrando a equação de governo (2) sob as hipóteses de que o termo fonte é distribuído uniformemente em cada volume de controle de comprimento $\mathrm{Dx}_{\mathrm{i}}$ e que os fluxos são distribuídos uniformemente nas interfaces dos volumes de controle, como mostrado na Figura 1 obtém-se, pelo método dos volumes finitos, a expressão (4):

$$
J_{i+1}-J_{i}=\left(S c_{i}+S p_{i} \cdot \phi_{i}\right) \cdot \Delta x_{i}
$$

A variável dependente $f_{i}$ a ser calculada quando da simulação, é posicionada no centro do volume de controle correspondente, em $x_{i}=D x_{i} / 2$. O esquema Flux-Spline assume que o fluxo em cada volume de controle varia linearmente, enquanto no esquema tradicional o fluxo é assumido, ou seja, $J_{i}=A_{i} . x_{i}+B_{i}$ sendo $A_{i}=\left(J_{i+1}-J_{i}\right) / D x_{i}$ e $B_{i}=J_{i}$. Substituindose esses valores na Eq. (3) e, após integração, impondo-se a

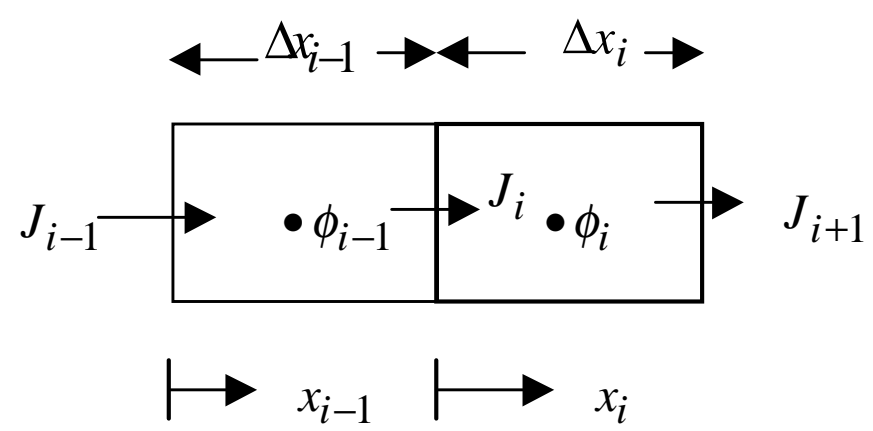

Figura 1. Discretização para volumes finitos

condição $f\left(x_{i}=D x_{i} / 2\right)=f_{i}$ no volume de controle i, obtém-se:

$$
\phi=\phi_{i}-\frac{\left(J_{i+1}-J_{i}\right)}{2} \cdot \frac{\Delta x_{i}}{\mathrm{~K}_{i}}\left(\frac{x_{i}^{2}}{\Delta x_{i}^{2}}-\frac{1}{4}\right)-J_{i} \cdot \frac{\Delta x_{i}}{K_{i}} \cdot\left(\frac{x_{i}}{\Delta x_{i}}-\frac{1}{2}\right)
$$

Note-se que $\left(J_{i+1}-J_{i}\right)$ aparece no termo quadrático da Eq. (5) num caso unidimensional, de acordo com a Eq. (4) representando a influência do termo fonte em cada volume de controle. Para um caso multidimensional se incluiria, também, a influência das outras direções pois, usando-se Eq. (1) mostra-se que:

$$
\frac{\partial J x}{\partial x}=S^{\phi}-\left(\frac{\partial J y}{\partial y}+\frac{\partial J z}{\partial z}\right)
$$

Assim, embora o perfil interpolante tenha sido derivado a partir da hipótese de unidimensionalidade, casos multidimensionais podem ser tratados de forma adequada e eficiente, devido a esta característica particular do esquema FluxSpline.

Dois volumes de controle adjacentes, como mostrado na Figura 1 possuem o mesmo valor de $f$ na interface comum. Assim, com $f\left(x_{i}=0\right)=f\left(x_{i-1}=D x_{i-1}\right)$ por meio da Eq. (5) obtém-se a equação para os fluxos na direção coordenada:

$$
J_{i}=J h a t_{i}+D J_{i} \cdot\left(\phi_{i-1}-\phi_{i}\right)
$$

em que:

$$
\begin{gathered}
D J_{i}=\frac{2.0}{\left(\frac{\Delta x_{i-1}}{K_{i-1}}+\frac{\Delta x_{i}}{K_{i}}\right)} \\
\text { hhat }_{i}=B J_{i} \cdot\left(J_{i}-J_{i+1}\right)+C J_{i} \cdot\left(J_{i}-J_{i-1}\right) \\
B J_{i}=D J_{i} \cdot \frac{\Delta x_{i}}{K_{i}} \cdot \frac{1}{8} \text { e } C J_{i}=D J_{i} \cdot \frac{\Delta x_{i-1}}{K_{i-1}} \cdot \frac{1}{8}
\end{gathered}
$$

Note-se que, como os fluxos são armazenados nas interfaces e assumidos com variação linear dentro dos volumes de controle, é assegurada a continuidade da derivada If $/ \mathbb{I} x$ ao longo de todo o domínio. A Eq. (7) quando substituída na equação de governo integrada (4) fornece a equação de discretização da variável dependente, representada por:

$$
\begin{aligned}
& A P_{i} \cdot \phi_{i}=A I M_{i} \cdot \phi_{i-1}+A I P_{i+1} \cdot \phi_{i+1}+S c_{i} \cdot \Delta x_{i}+S s p_{i} \\
& \text { em que, }
\end{aligned}
$$

$$
A I M_{i}=D J_{i+1} \quad \text { e } \quad A I P_{i}=D J_{i}
$$




$$
\begin{gathered}
A P_{i}=A I M_{i}+A I P_{i}-S p_{i} \cdot \Delta x_{i} \\
S s p_{i}=\left(\text { Jhat }_{i}-J_{h a t_{i+1}}\right)
\end{gathered}
$$

O método de solução empregado consiste em arbitrar, inicialmente, uma distribuição para a variável dependente e calcular os fluxos que são então usados para obter-se o termo fonte adicional Ssp, característico do esquema Flux-Spline. O sistema de equações algébricas para a variável dependente forma uma matriz tridiagonal, que é então resolvida usando-se o algoritmo TDMA (Tridiagonal-Matrix Algorithm) como mostrado em Patankar (1980). Com esses valores retorna-se ao cálculo dos fluxos até a obtenção da convergência da variável dependente e um resíduo aceitável para a equação de governo. O erro percentual cometido na simulação é definido para cada ponto do domínio, na forma:

$$
\text { Erro }=100 \cdot a b s\left(1-\frac{\phi_{\text {num }}}{\phi_{\text {exato }}}\right)
$$

em que $f_{\text {num }}$ é o valor da variável dependente obtida pelo procedimento numérico e $f_{\text {exato }} \mathrm{O}$ valor obtido pelo procedimento analítico. Será usado, para a construção do gráfico de erro, o valor do máximo erro cometido no domínio de cálculo como, função de uma malha regular com $\mathrm{n}$ volumes de controle em cada direção coordenada, de forma a comparar a ordem de precisão dos esquemas de discretização.

\section{Difusão Multidimensional}

Integrando-se, de acordo com o método de volumes finitos, a Eq. (1) e aplicando, então, a expressão desenvolvida para o fluxo na direção $x$, às outras direções coordenadas, obtém-se as equações de discretização para os fluxos em x, y e z e para a variável dependente. As equações são:

$$
A P_{i, j, k} \cdot \phi_{i, j, k}=\sum A n b_{i, j, k} \cdot \phi n b_{i, j, k}+S c_{i, j, k} \cdot \Delta x_{i} \cdot \Delta y_{j} \cdot \Delta z_{k}+S s p_{i, j, k}
$$

em que,

$$
\begin{aligned}
& A P_{i, j, k}=A I M_{i, j, k}+ A I P_{i, j, k}+A J M_{i, j, k}+A J P_{i, j, k}+A k M_{i, j, k}+A k P_{i, j, k}- \\
& S p_{i, j, k} \cdot \Delta x_{i} \cdot \Delta y_{j} \cdot \Delta z_{k} \\
& \sum A n b_{i, j, k} \cdot \phi n b_{i, j, k}= A I M_{i, j, k} \cdot \phi_{i-1, j, k}+A I P_{i, j, k} \cdot \phi_{i+1, j, k}+A J M_{i, j, k} \cdot \phi_{i, j-1, k}+ \\
& A J P_{i, j, k} \cdot \phi_{i, j+1, k}+A K M_{i, j, k} \cdot \phi_{i, j, k-1}+A K P_{i, j, k} \cdot \phi_{i, j, k+1} \\
& S S p_{i, j, k}=\left(J h a t X_{i, j, k}-J h a t X_{i+1, j, k}\right) \cdot \Delta y_{j} \cdot \Delta z_{k}+ \\
&\left(J h a t Y_{i, j, k}-J h a t Y_{i, j+1, k}\right) \cdot \Delta x_{i} \cdot \Delta z_{k}+ \\
&\left(J h a t Z_{i, j, k}-J h a t Z_{i, j, k+1}\right) \cdot \Delta x_{i} \cdot \Delta y_{j} \\
& A I M_{i, j, k}=D J X_{i, j, k} \cdot \Delta y_{j} \cdot \Delta z_{k} \\
& A I P_{i, j, k}= D J X_{i+1, j, k} \cdot \Delta y_{j} \cdot \Delta z_{k} \\
& A J M_{i, j, k}=D J Y_{i, j, k} \cdot \Delta x_{i} \cdot \Delta z_{k} \\
& A J P_{i, j, k}=D J Y_{i, j+1, k} \cdot \Delta x_{i} \cdot \Delta z_{k} \\
& A K M_{i, j, k}=D J Z_{i, j, k} \cdot \Delta x_{i} \cdot \Delta y_{j}
\end{aligned}
$$

$$
A K P_{i, j, k}=D J Z_{i, j, k+1} \cdot \Delta x_{i} \cdot \Delta y_{j}
$$

As expressões para os fluxos $J X, J Y, J Z$, os pseudo-fluxos Jhat $X$, JhatY e JhatZ e os demais coeficientes, DJX, BJX, CJX, $D J Y, B J Y, C J Y, D J Z, B J Z$ e $C J Z$, são análogas àquelas deduzidas para o caso unidimensional. $\mathrm{O}$ método de solução para casos tridimensionais é similar ao utilizado no caso unidimensional, sendo que na resolução do sistema de equações algébricas é usado o método Line-by-Line, descrito por Patankar (1980) em cada plano coordenado nas direções $\mathrm{x}, \mathrm{y}$ e $\mathrm{z}$.

\section{RESULTADOS E DISCUSSÃO}

\section{Aplicação do esquema a problemas-teste}

A distribuição da variável dependente $f$, num domínio adimensional entre zero e um para todos os eixos coordenados, com o coeficiente de difusão $\mathrm{K}$ assumido como constante, obedece às seguintes distribuições adimensionais:

Caso 1:

$$
F 1(X, Y, Z)=\exp (X) \cdot \exp (Y) \cdot \exp (Z)
$$

Substituindo-se essa distribuição na equação de governo (1) obtém-se a distribuição dos termos-fonte que, para este caso, é fornecida pela expressão:

$$
S^{F 1}=-3 \cdot F 1
$$

Caso 2:

$$
F 2(X, Y, Z)=\sin \left(\frac{\pi \cdot X}{2}\right) \cdot \cos \left(\frac{\pi \cdot Y}{2}\right) \cdot \exp (Z)
$$

Da mesma forma, obtém-se a distribuição dos termos-fonte que, neste caso, é:

$$
S^{F 2}=\left(\frac{\pi^{2}}{2}-1\right) \cdot F 2
$$

A curva representando o erro percentual máximo, função de uma malha com $\mathrm{N}$ volumes de controle, em cada direção, para os dois casos, está plotada na Figura 2.

Como mostrado graficamente para os casos tratados, o esquema Flux-Spline necessita para determinado nível de erro, de malhas menos refinadas que o tradicional esquema de diferenças centrais. A razão disto, é o perfil interpolante característico do esquema descrito pela Eq. (5) cujo termo quadrático, que contém a variação do fluxo ao longo do volume de controle promove, ao mesmo tempo, o tratamento do termo fonte e da influência das outras dimensões. Esta característica fornece ao esquema maior precisão, possibilitando menor esforço computacional ao se simular problemas onde seja necessário abordar o comportamento transiente dos fenômenos. Para problemas envolvendo escoamentos como os que ocorrem no transporte de calor e massa durante a secagem e/ou armazenamento de grãos, as equações de governo possuem, além do termo difusivo, termos de transporte convectivo, devido ao campo de velocidades. Assim, o perfil interpolante a ser gerado pelo esquema é mais complexo que o parabólico aqui derivado para difusão pura, sendo composto por uma reta mais um termo exponencial. 


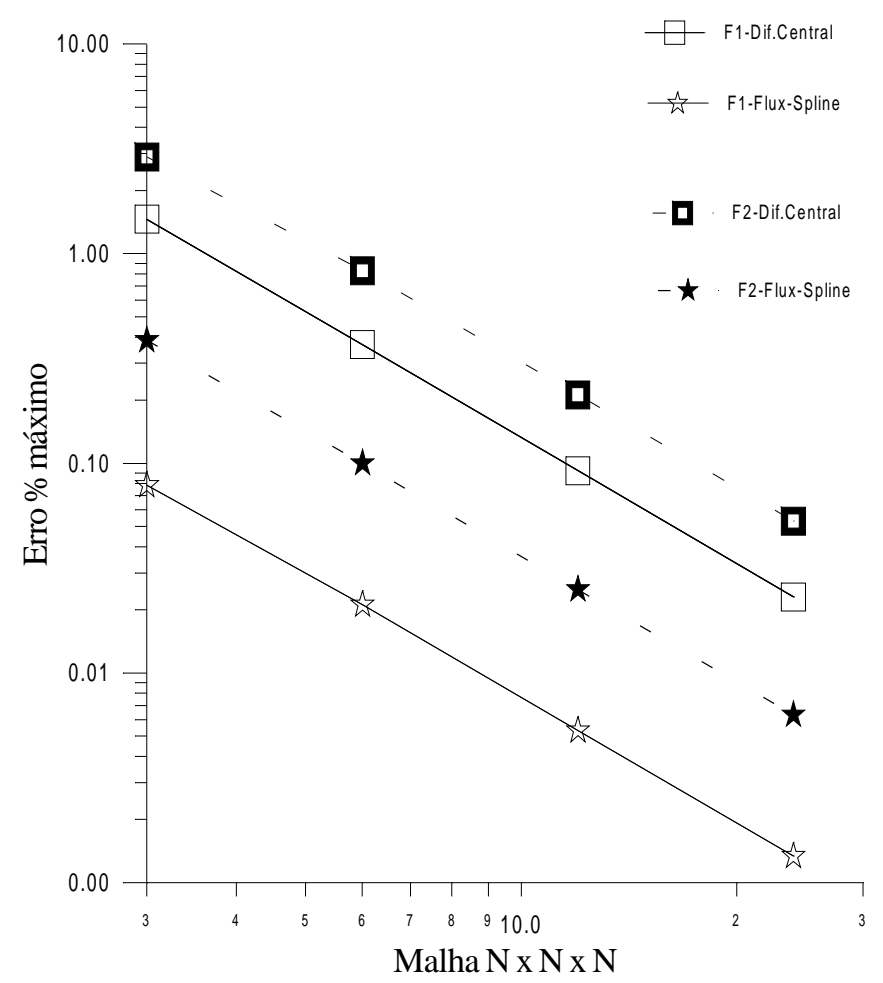

Figura 2. Erro como função da malha

\section{CONCLUSÕES}

Com base nas discussões referentes aos resultados obtidos neste trabalho, pode-se concluir que:

1. O esquema Flux-Spline mostrou-se, em termos de erro, mais preciso que o tradicional esquema de diferença central, para problemas difusivos tridimensionais em regime permanente.

2. Ao se fixar certo nível de erro, a malha empregada para alcançá-lo utilizando-se o esquema proposto, envolve um número significativamente menor de volumes de controle que o empregado pelo esquema de diferença central.
3. A utilização do esquema Flux-Spline em problemas difusivos tridimensionais transientes, pode ser recomendada com a finalidade de se diminuir o esforço computacional.

\section{REFERÊNCIAS BIBLIOGRÁFICAS}

FRANÇA, A.S.; FORTES, M. Simulação bidimensional de secadores de grãos. In: SIMPÓSIO MINEIRO DE MECÂNICA COMPUTACIONAL, 1991, Belo Horizonte. Anais... Belo Horizonte: UFMG, 1991. p.518-527.

NIECKELE, A.O. Development and evaluation of numerical schemes for the solution of convection-diffusion equations. Minnesota, USA: University of Minnesota, 1985, 296p. Ph.D. Thesis.

OLIVEIRA, L.S.; FORTES, M.; FERREIRA, W.R. Natural drying of a vertical slab of a biological material. In: SIMPÓSIO MINEIRO DE MECÂNICA COMPUTACIONAL, 1991, Belo Horizonte. Anais... Belo Horizonte: UFMG, 1991. p.286-294.

OLIVEIRA, P.C. Esquema FLUX-SPLINE aplicado em cavidades abertas com convecção natural. Campinas: UNICAMP, 1997. 196p. Tese Doutorado

PATANKAR, S.V. Numerical heat transfer and fluid flow. New York: Hemisphere Publishing Corporation, 1980.197 p.

VAREJÃO, L.M.C. Flux-Spline method for heat, mass and momentum transfer Minnesota, USA: University of Minnesota, 1979. 235p. Ph. D. Thesis.

VAREJÃO, L.M.C.; PATANKAR, S.V.; KARKI, K.C. Flux-Spline method for convection-diffusion problems. In: SIMPÓSIO MINEIRO DE MECÂNICA COMPUTACIONAL, 1991, Belo Horizonte. Anais... Belo Horizonte: UFMG, 1991a. p.161-174.

VAREJÃO, L.M.C.; PATANKAR, S.V.; KARKI, K.C. Flux-Spline method for conduction-type problems. In: SIMPÓSIO MINEIRODE MECÂNICA COMPUTACIONAL, 1991, Belo Horizonte. Anais... Belo Horizonte: UFMG, 1991b. p. 175-184. 